\title{
BACKPACK MOBILE MAPPING SOLUTION FOR DTM EXTRACTION OF LARGE INACCESSIBLE SPACES
}

\author{
F. Fassi a ${ }^{*}$, L. Perfetti a \\ a 3D Survey Group, Dept. of Architecture, Built environment and Construction engineering (ABC), Politecnico di Milano, Italy
} (francesco.fassi, luca.perfetti)@polimi.it

Commission II, WG II/8

KEY WORDS: DTM, mobile mapping systems, backpack

\begin{abstract}
:
The paper presents the case study of the complete 3D survey of the area of the Fort of Pietole in Borgo Virgilio using the Leica Pegasus Backpack wearable Mobile Mapping System (MMS). Surveying the site is challenging because of its complex topology on the one hand (with notably narrow passages) and because of the presence of vegetation on the other. The framework within which this research takes place is the Fort of Pietole survey project that aims at the extraction of the Digital Terrain Model (DTM) of the area and the georeferencing of the fort defensive structures. The requirement of the project is the $3 \mathrm{D}$ reconstruction of the whole area at an accuracy that stands between a big scale environmental survey and a small-scale architectonic survey (1:500).

The project is the opportunity to discuss the state of the art of wearable MMS, and to test the versatility and accuracy outcomes of the Pegasus Backpack under varying and challenging condition (indoor-outdoor, even-uneven pavement, satellite covered-denied areas) with the ambitious goal to use only the backpack MMS to record all the data from the DTM to the indoor narrow structures.
\end{abstract}

\section{INTRODUCTION}

\subsection{The fortress of Pietole}

The Fort of Pietole is a military fort constructed at the beginning of the XIX century. It is located in the south-eastern outskirts of the city of Mantua, in the municipality of Borgo Virgilio in the "Vecchia Pietole," on the right bank of the Mincio river on a slight hill (Virgil's Hill) where it is believed to be located the birthplace of the Latin poet.

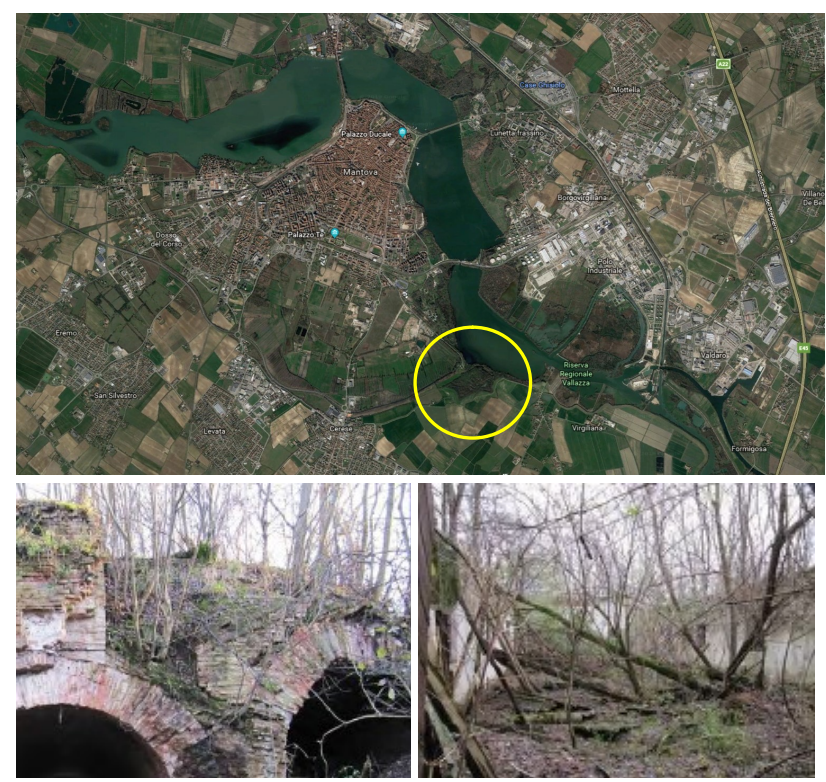

Figure 1. Location of the Fort of Pietole at the south of Mantua (top). State of neglect of the defensive structures and vegetation grow (bottom).

The site is surrounded by cultivated field and natural and anthropic areas of high environmental and landscape value. The fort itself encloses a strong cultural heritage value, the southern development of the fort is preserved in the same anthropization conditions of the early nineteenth century and this confers to the whole structure a peculiar and rare value to be found among the defensive fortresses of other Italian cities that have been instead transformed or incorporated in the modern urbanization.

The Fort of Pietole can be classified as an "asymmetric star fort," and it is a typical example of the French fortified architecture. It covers an area of around $330,000 \mathrm{~m}^{2}$ in open meadowland.

The fort is structured in many fortification levels, the focus of this test is the central core and a portion of the second level composed of large terraces and the moat. The core consist of a vast parade ground protected by the bastion's which sides houses the artillery defensive structures, the "case matte," used to overlook the second level to protect the moat, that was flooded only in the event of war, which directly bathes the bastion's walls covered in bricks.

The fort of Pietole has been used and transformed several times during its life span and today is in a state of neglect. At present days, uncontrolled vegetation has grown back and spread to cover all the fort extension with trees and bushes making it difficult to access the area (Figure 1).

\subsection{The project}

The municipality of Borgo Virgilio together with the Politecnico di Milano is designing the requalification of the area to make the fort accessible to the public, to valorize and to revive the great value of the area both from the architectonic conservation point of view and from the environmental one. The project aims to rethink the Fort area as a connection between the old town, the lakes and the protected natural park of the Mincio River enhancing the tourist attractiveness through the creation of a range of services and facilities. Increased accessibility (by land and by water), the creation of new spaces (indoor and outdoor) and the design of the conservation 
intervention (choice of materials and construction technologies to be employed) are some of the main topics of the project.

It is, therefore, clear as a deep knowledge of the fortress to identify its specificity and its tangible and intangible heritage is crucial to strengthen its identity and enhance its values through a conscious approach.

Before the definition of the conservation and valorization project, understanding the area in mandatory, a complete $3 \mathrm{D}$ survey is a preferable way to describe in depth both the territory morphology and the architectural layout.

\subsection{The survey area}

The focus of the survey is dual: environmental to produce and to understand at big scale the overall DTM of the fort area, and architectonic at the middle or little scale to get the position of the ruins and buildings in the area and to understand the planaltimetric trend of underground structures.

The core area, the object of this study, measure around 600 meter in length and around 220 hectares of surface. The core area is delimited by tall fortifications on the east, south, and west sides. Along this fortified perimeter, partially incorporated in the terrain morphology, seven buildings overlook the lower fortification level through slit openings. The architecture of the fort also consists of narrow passages connecting the structures of the fort. Finally, in the center of the fort, enclosed by the fortifications there is a large embankment forming the complex digital terrain model (DTM) that at date is covered in vegetation.

\subsection{The survey methods the state of the art}

A DTM can be obtained by different means and approaches, the survey project of the Fort of Pietole required the survey of the terrain surface as well as of the fort's buildings, geometry, and indoor spaces. Moreover, the resolution of both must suit the big architectonic scale (1:200 - 1:500).

Range-based techniques are usually the choice for the DTM extraction; aerial lidar acquisitions are typically employed.

A technique that uses an active optical sensor is to be preferred to others based on passive optical sensors. Independently on the resolution, lidar sensors allow points to be measured on the ground by "penetrating" the vegetation; conversely, photogrammetry is bound to the sampling resolution to distinguish details and would require an extremely highresolution in other to reconstruct even few points on the ground through the bushes. For this reason, if the aim is to obtain the terrain model - where relative sparse points are sufficient - a range-based approach allows acquiring a low number of points while still be able to register the terrain surface.

An aerial approach was rejected for the following motivation: i) a classical lidar acquisition is expensive and provides a too lowresolution point cloud that would not meet the project requirements, i.e., an architectonic scale survey. ii) A lidar multi-rotor UAV (Unmanned Aerial Vehicle) approach, although would meet the resolution requirements, it too is an expensive technology. iii) A top-down nadiral acquisition geometry is not the ideal one to survey the architectonic structures together with the DTM.

Based on the requirements of the case study, an interesting strategy was to employ a range-based mobile mapping system at ground level. Nowadays MMSs are growing fast and improving to the point that new applications are possible. Such devises can be suited for a large range of scenarios and potentially can be used for big scale architectonic survey of the territory and of architecture as well.
An MMS is based on the possibility of associate 3D data acquired by a mapping sensor together with the information on the position and orientation of the sensor itself. They are therefore composed of three main components: a mapping sensor, a positioning or many positioning devices and a control unit able to synchronize the two (Puente et al. 2013; Toschi et al. 2015).

Their accuracy class depends greatly on these three components, and different types of MMSs can house different types of sensors. MMSs can be divided in vehicle mounted, trolley mounted or wearable.

1.4.1 Vehicle mounted MMSs: are already state of the art and are commonly used for urban mapping, their accuracy class can be very high mainly due to their size that makes it possible to house high-grade sensors and to the possibility to use wheel odometry and high-quality GNSS receiver.

1.4.2 Trolley mounted MMSs: are a step down in accuracy but a step up in mobility, they require, like vehicle MMSs, even pavements and can house good quality sensor as well. Trolleys can also be used in indoor environments at the expense of GNSS signal and accuracy.

1.4.3 Wearable MMSs: are the real novelty nowadays, they can be backpack mounted or handheld devices and are generally characterized by lower-grade sensors and positioning units. They offer the advantage of being employable on uneven pavements, stairs, and so forth. Wearable MMSs are very promising to simplify the mapping process of complex environments ranging from outdoor to indoor to narrow areas. Some features GNSS receiver that can minimize drift error outdoor while they use Simultaneous Localisation And Mapping (SLAM) algorithms indoor (Thomson et al. 2013; Lauterbach et al. 2015).

The development and working scenarios of wearable MMSs are of great interest, especially the development of SLAM-based MMSs for their use indoor or in GNSS denied environments. Their accuracy, however, needs to be evaluated and tested (Thomson et al. 2013; Nocerino et al. 2017; Mandelli et al. 2017; Lehntola et al. 2017; Tucci et al. 2017; Masiero et al. 2018; Sammartano et al. 2018).

The Fort of Pietole and the requirements of the project offered the possibility to test the use of a mobile mapping system to significantly ease the mapping process. The chosen MMS for this test is the Leica Pegasus Backpack.

Our interest is the evaluation of the geometric accuracy that can be achieved in such a complex scenario, however wearable MMS are also of interest in the field of forest analysis where they were successfully employed for forest inventory survey applications showing an advantage against traditional TLSs (Terrestrial Laser Scanners) in terms of speed of the acquisition and data completeness by allowing a mobile surveys and by avoiding shadow areas (Liang et al. 2016; Ryding et al. 2015). The same advantages can be observed for architecture 3D mapping (Mandelli et al. 2017).

\section{THE SURVEY}

\subsection{A mobile mapping challenge}

The Fort of Pietole as a case study is a real challenge and therefore offers many opportunities to evaluate the performance of the backpack MMS. Among its extension the fort features: i) open areas, ii) GNSS denied outdoor areas, iii) indoor environments and iv) narrow tunnels.

Along the acquisition path designed to map the whole fort, the conditions change drastically: the acquisition started outdoor at the center of the fort in open space under a strong GNSS signal 
(Figure 2), here the operator performed the instrument calibration to ensure good global positioning. It then required to move under the canopy were the GNSS got intermitting and then lost in some portion (the survey has been carried out in February). The ground also changed between being flat and easily walkable to being quite rough while walking out from the pathway and into the bushes under denser vegetation (Figure 2), here the walking path had been cleared and prepared in real time just before the operator to follow.

The fortified structure of the fort offered the opportunity to test both the SLAM capability of the tested device and the possibility of frequently switching between indoor and outdoor areas. The structures of the fort can be divided in i) case matte, large structures that required to follow a long indoor acquisition path to be surveyed, ii) passages, narrow and long corridors that allowed to test the SLAM drift and iii) small guerite composed of a single small room.

The change in path roughness, vegetation cover and the alternation between indoor and outdoor environment make this case study extremely challenging and realistic at the same time, suited to stress the limitations of the employed backpack solution and different acquisition strategy as well.

\subsection{The Leica Pegasus Backpack}

The Leica Pegasus Backpack is a wearable mobile mapping system designed to be used outdoors in the presence of a strong GNSS signal that is important to constrain the system trajectory within the positioning system accuracy at each moment of the acquisition. However, the Pegasus backpack can also be used in the lack of GNSS aid; in this case, the trajectory computation relies on SLAM algorithms. The backpack MMS was chosen as a time-effective alternative to other more traditional solutions, its maneuverability allows it to be carried around by a single operator simply walking along the area to be recorded on uneven terrain too. The GNSS receiver promise to contain the drift error of a long acquisition path even if under varying obstruction condition.

The possibility to use the Pegasus Backpack on rough pavement and the use of both the GNSS receiver and the SLAM aid makes this solution extraordinarily versatile and qualify it as a cost- and time- effective alternative to acquire the complete $3 \mathrm{D}$ survey of the fort using one instrument only.
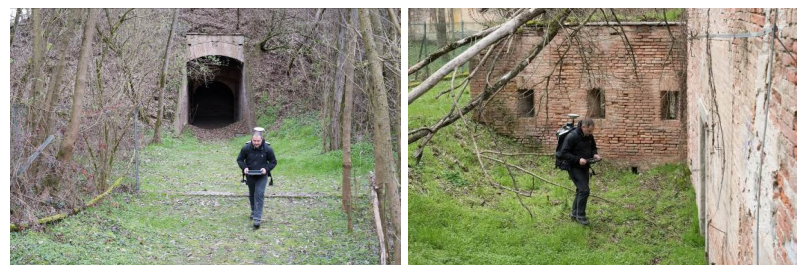

Figure 2. Leica personnel scanning the fort with the Pegasus Backpack.

The Pegasus backpack features two Velodyne profilometers one mounted to acquire data horizontally, and one mounted to acquire vertically few degrees off the vertical axis and five cameras (2048 x 2048 pixel each) coupled with fisheye lenses used to colorize the resulting point cloud. The horizontal Velodyne was used in the SLAM data processing only while the second sensor is used to produce the final registered point cloud. The accuracy of the sensor is of $\pm 3 \mathrm{~cm}$ that suits perfectly the application since the aim is to obtain a dense big scale point-cloud to serve as a base for the little scale survey to follow.
The point cloud produced by the vertically mounted profilometer produced complete results describing the geometry of the structures from the ground to building's eaves.
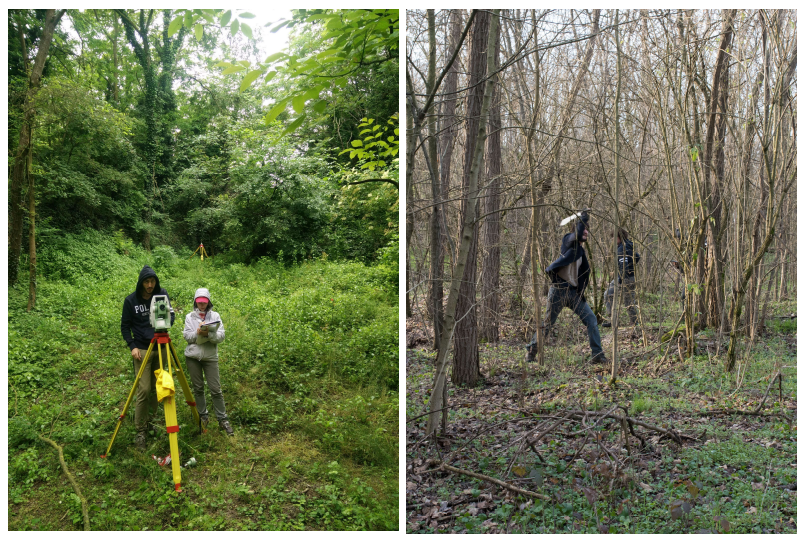

Figure 3. Some phases of the total station survey and the quick cleaning of the path before the backpack walk.

\subsection{The acquisitions}

Two separate acquisitions were carried out separately to limit the survey time of each path and to better organize the activity on site.

i) The first acquisition was meat to survey the whole area of the fort that was better accessible i.e., the walk followed the pathways, comprehended all the architectonic structure and some portion characterized by a rough terrain under the wood that required to be cleared of the bushes in real-time before the operator could pass (Figure 2).
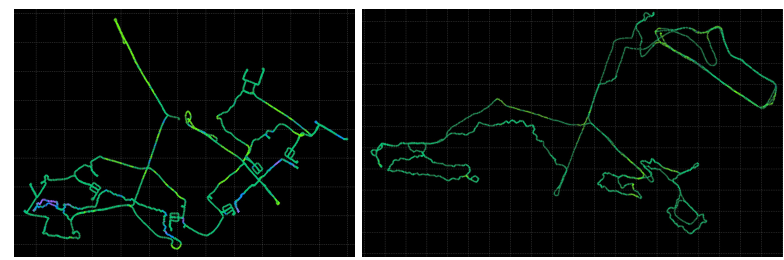

Figure 3 . The two acquisition paths that are necessary to cover the whole area.

ii) The second acquisition covered the steeper portion of the terrain under the wood that not only required to be cleared from vegetation, but that was also challenging because of the vibrations transferred to the backpack during the walk.

The decision to separate the two acquisition also raises with the idea of separating the most environmentally challenging acquisition from the main path. Both the acquisition started and ended at the same point where the GNSS initialization took also place at the very beginning and end of each path.

The paths for the two main acquisitions have been decided on site after some test and trials performed on the first day.

For all the "case matte," the defensive structures of the fort, that are based on the same layout, a precise indoor path has been defined to reduce the length of the indoor acquisition that would rely solely on SLAM.

A test has also been carried out regarding the small guardroom structure distributed around the fort to decide whether to enter and survey them during the main acquisition or whether to ignore them to survey the indoor space later. The walk was interrupted at each guardroom to acquire the small indoor space; this approach has been soon discarded since it has been observed that each switch between indoor and outdoor 
environment produces a week connection that could cause misalignments. For this reason, to avoid possible errors, it has been decided to limit these week connections to the minimum needed.

A test has also been performed to try changing the acquisition velocity from a regular/slow walk to a fast walk, real-time feedback from the instrument suggested the first approach to be preferable. Moreover, many of the areas could be surveyed without caution.

All the data acquisitions have been carried out by specialized Leica personnel and spanned through two days on site.

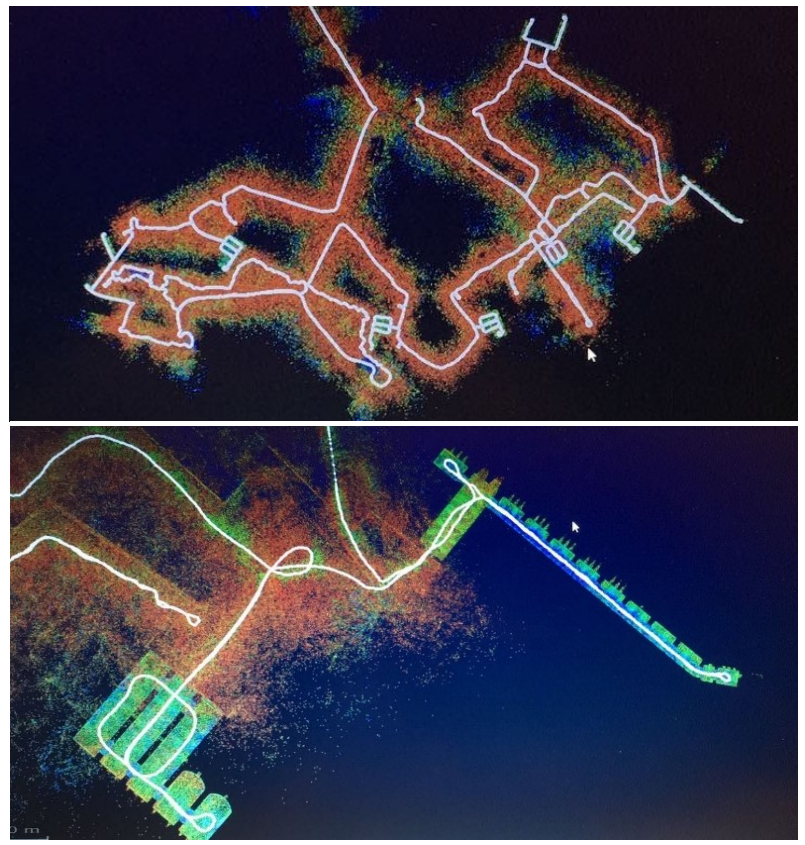

Figure 4. Views of the Pegasus Backpack registered point cloud and of the adjusted trajectory followed during the acquisition.

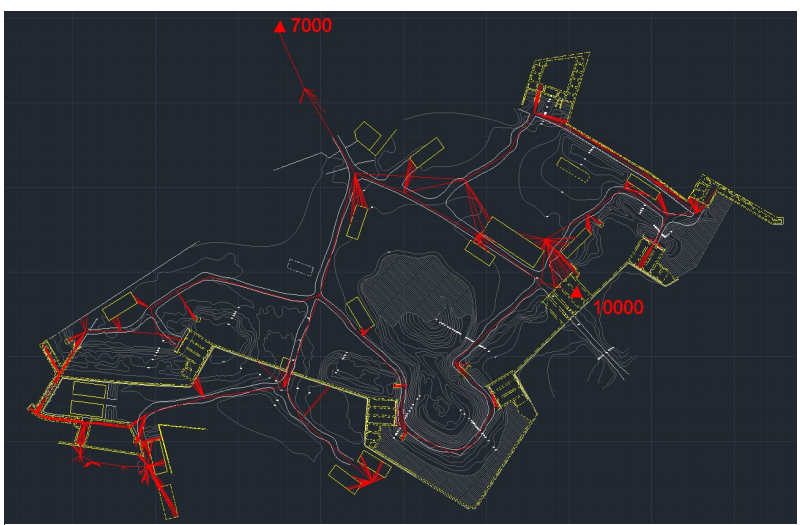

Figure 5. topographic network (redlines). The point 7000 and 10000 are the two point for the coordinate system transformation.

\subsection{Data validation}

Conceptually, a topographic survey is not indispensable for this type of activity, indeed the aim behind performing the survey with a mobile scanner solution is precisely to acquire the complete geometry of large areas without having to rely on the classical topographic acquisition which, as in this case, is complex, time-consuming, sometimes dangerous and generally not exhaustive in terms of completeness of the data.
The collection of GCPs, here, had the double goal to test the final accuracy of the Pegasus Backpack and as support during the elaboration of the trajectory in case of poor satellite coverage.

The survey was conducted in two days of work, from the laying out of temporary vertices on the ground to the measurements of the GCPs, the checkpoints and the network itself.

The network is composed of three closed loops that were planned to reach all the main parts of the area with the idea to collect an architectonic point on the buildings that could be visible in the point cloud. A total number of 27 stations were used to collect around 900 point. Some of them are architectonic points (around 500) to be used as GCP inside the process, the others are points that lay on the structures (around 400) to be used in a second phase as checkpoints to verify the planar position and orientation of buildings wall and other structures. Two of the network vertices positioned at the boundaries of the fort area outside the vegetation were used to locate the local network in the WGS84 coordinate system. For this reason, a 5-hour static acquisition was performed on both those points.

The RMS on the network points is about $7 \mathrm{~mm}$.

\section{DATA ELABORATION}

At the time of elaboration (2018), the elaboration of the Pegasus Backpack data was a burdensome process that required specific training with different software module to complete the whole elaboration. With the new software releases, everything (trajectory processing and cloud processing) has been incorporated in Pegasus Manager.

The elaboration pipeline is divided into two main steps covering the processing of the three positioning resources for trajectory computation (GNSS, IMU, and SLAM) that the Pegasus Backpack exploits.

The first step, covered by the "Inertial Explorer software," is to compute the trajectory of the acquisition integrating into the adjustment GNSS Data, Inertial data and, eventually, additional information such as measured GCPs and tie points.

The second step, covered by the "SLAM module," is to adjust the previous elaboration by computing a SLAM optimization. The optimization can be applied to the whole trajectory or a specific segment.

\subsection{The elaboration of Pietole Data}

The first step of the elaboration is to statistically calculate the best trajectory analyzing GNSS and Inertial data.

To geolocate the Pietole survey, the GNSS data were elaborated in ARTK (Advanced Real-Time Kinematic) post-processing using the "Acquanegra" GNSS station as reference fixed master. This process is necessary for outdoor surveys and to georeferencing the data in geographical coordinates; without a GNSS master station, likewise, for pure indoor application, the backpack acquisition results in the local coordinate system.

Eventually, a user GNSS static acquisition on a known coordinates point can be used. The Pegasus Backpack can work in RTK (Real-Time Kinematic) as well. The coordinate system used for the Pietole case study is WGS84.

Under the vegetation, even if in winter with no foliage, the GNSS data resulted in being poor and not optimal. To consider more satellite a less rigorous, more tolerant approach was adopted, that means that less restrictive parameters were used: a cut-off angle of $8^{\circ}$ (normally is $12^{\circ}$ ) and GNSS data up to PDOP (Position Dilution of Precision) 5 was accepted. 
The method used to analyse the trajectory is a "tightly coupled methods" with an iterative approach that means that GNSS and INS (Inertial Navigation System) data are processed simultaneously and that the trajectory is analyzed three times forward and three times reversed, the computation is accepted when the ambiguity is resolved in both directions of navigation. The evaluation of the quality of the trajectory must be computed in two steps. The first is to evaluate the statistical report of the trajectory adjustment in Inertial Explorer, and the second is to visually inspect the processed point cloud via slicing. Regarding the first step, In the project, the following aspects ware taken into consideration (Figure 6):

1. Statistical estimation of position accuracy, the graph shows the accuracy of the relative position considering all the GNSS influences and errors, such as the satellite constellation, PDOP, atmospheric influences, etc.... It provides a good indication of the accuracy of the expected trajectory position. Without reflecting any systematic error, such as errors in the antenna height, coordinates errors of the reference station or any coordinate transformation error.

2. Forward/reverse combined separation, the graph shows the position difference in $\mathrm{E}, \mathrm{N}$, and Q, i.e., the deviation between the result of the forward and the reverse processing. In good acquisitions, the expected variation along the trajectory should be around $\leq \pm 10$ $\mathrm{cm}$. This graph can be very useful to find where it is likely to get misalignments in the point cloud and therefore, if required, to impose more constraints such as GCPS.

3. The float/fixed ambiguity graph shows the quality of the GNSS signal: whether the trajectory is fixed, well constrained (green); whether it is fixed in only one direction (blue or light blue), trajectory can be improved; and whether it is not fixed, it is "floating" (red), in this case, only the IMU data is taken into consideration for the prediction of the next position and the error in absolute precision will not be controllable.

4. The last graph shows the differences in the attitude of the mobile antenna between the forward and the reverse elaboration separating the three-rotation component of roll pitch and heading. It is measured in arcmin. In a good trajectory estimation, the values of pitch and roll should be near 0,1-0,2 arcmin and 0,4 0,5 for the heading deviations.

5. The number of satellites visible during the acquisition, At least five satellites are needed from the same constellation to count as a constraint over the trajectory (fixed position).

The trajectory was computed several times, comparing the different results manually with the help of the described graphs to choose the best statistical solution.

After having obtained the best possible elaboration of the trajectory, the second validation step was to manually verify the point cloud to identify possible misalignment. This manual check is done by slicing the point cloud in different positions starting, paraphs, from the point cloud area corresponding to the peaks in graph 2 and the graph 4 (Figure 7). for these positions, if a misalignment is detected, it is possible to add additional constraints such as GCPs and tie points to further improve the result.

The case study characteristic, as expected, posed great challenges over the trajectory computation, it was therefore mandatory to adjust the initial result using additional constraints. As Figure 7 shows, on the left part of the graphs, we have a large portion of the acquisition with no GNSS signal (graph 3) with separation values in position between $+15 \mathrm{~m}$ and $-55 \mathrm{~m}$ (graph 2). Also, the value of attitude separation is much higher than acceptable (graph 4).

A total station survey was performed in order to collect enough GCPs evenly distributed in the survey area and a second computation of the trajectory was performed considering these points as constraints into the process. The positioning of GCPs is a manual operation that can be performed both on image and on the point cloud.

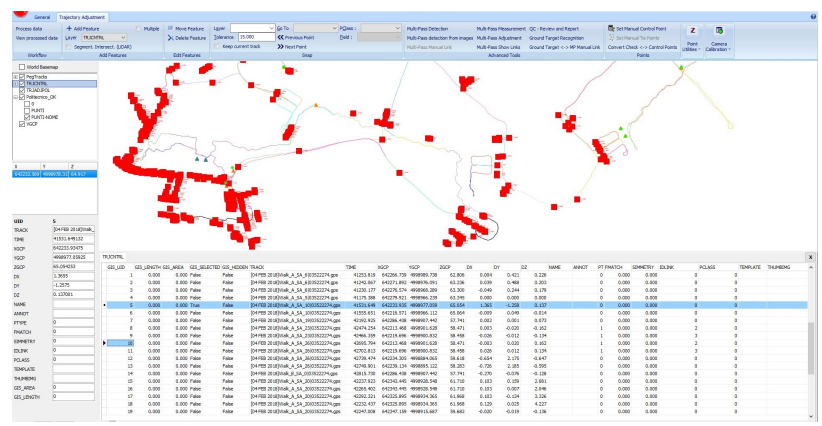

Figure 6. The map of GCPs inside Pegasus manager software after the last adjustment. The deviation is around $10-20 \mathrm{~cm}$, for few unresolved areas 1 meter.

Figure 7, offers a comparison of the two elaborations: on the left column, the result obtained from the first processing, only GNSS and IMU data were used; on the right column, the best statistical solution obtained with the aid of GCPs. It is evident that the separation in position (graph 2) has improved considerably with only one peak values between -4 and 7,5 m.

Once the best possible trajectory is obtained, it is possible to perform the second step of the elaboration, the SLAM optimization. The SLAM adjustment was performed only for the indoor architectonic structures and for the areas in front of the buildings to impose a constraint where there was a switch of environment between indoor and outdoor. SLAM calculation failed for the open spaces of the fort due to the moving vegetation.

\subsection{Manual refinements}

After the computation of the best trajectory and the creation of the point cloud, manual refinements followed to adjust the reconstruction of the unresolved portion of the trajectory (central-left part of graphs in Figure 7, right column). The point cloud has been imported in the software Leica Cyclone segmented in short walks; here the two acquisitions described in section 2.3 were merged and the small point cloud segments that showed misalignments were adjusted by means of cloud to cloud registration or discarded entirely if redundant.

The registration has been checked visually by slicing the point cloud and by using checkpoints measured with the total station to control the position and alignment of the architectonic structures.

Overall, final error remained within $50 \mathrm{~cm}$ deviation at most. 

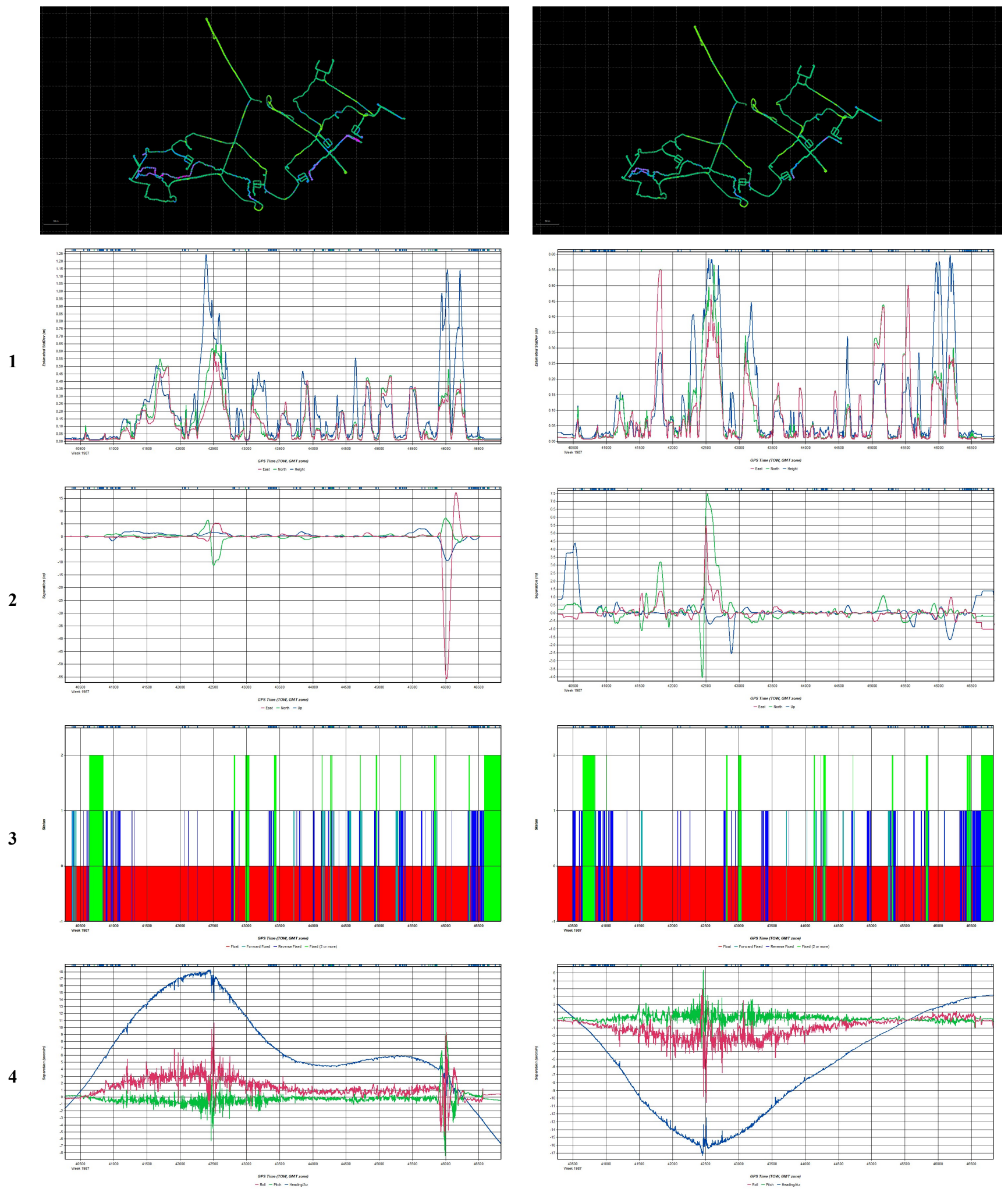

Figure 7. The graphs of the elaboration of the first trajectory. On the left column, the result of the tightly coupled process using only GNSS and IMU. The adjustment did not produce good results due to the poor or lacking GNSS signal (red areas in graph 3 ) here the estimation of the position accuracy (graph 2) along the trajectory has an estimated standard deviation lower than $0.5 \mathrm{~m}$ but with peaks of $1.25 \mathrm{~m}$ and a corresponding separation in E, N, Q position in Forward and reverse calculation of $15 \mathrm{~m}$ and $-55 \mathrm{~m}$. The correction with the GCP improved the result (right column), standard deviation lower than $0.6 \mathrm{~m}$, and separation in the position of about $\pm 1 \mathrm{~m}$. Only for the central part of the path, an unresolved portion of the trajectory required manual intervention. 


\section{OUTCOMES}

\subsection{Data Quality}

The point cloud produced by the backpack is complete in all the buildings parts, the use of the vertically positioned Velodyne allows the registration of complete cross-sections of the environment from the ground to the eaves without the need to rotate the instrument or to pivot with the body. Point cloud resolution, other than from the lidar sensor installed, depends both form the distance with the scanned surfaces and from the operator velocity. It has been observed to be always sufficient for big scale architectonic applications up to 1:100.

The noise of the point cloud is about $5-6 \mathrm{~cm}$, allowing the use of the Pegasus Backpack up to scale 1:200 (tolerance of $8 \mathrm{~cm}$ ) under ideal conditions.

The case study of the Fort of Pietole was extremely challenging mainly due to two reasons: i) the intermitting GNSS signal under the vegetation and ii) the lack of stable geometries in the woods that would have allowed a better performance of the SLAM as in the indoor environments. Under such conditions, the accuracy achievable by the backpack MMS although potentially high (1:200 based on the Velodyne specs) depends mainly on the success of the processing.

The test highlighted that in lack of significant scene geometry for the SLAM optimization to succeed, good GNSS signal is mandatory to rely on the MMS only. For the fort, the use during the elaboration process of GCPs measured with the total station was required to obtain satisfactory results.

The final accuracy of the produced point cloud, after the manual refinements (section 3.2), matched the architectonic scale of 1:500 and therefore met the requirements of the Pietole survey project.
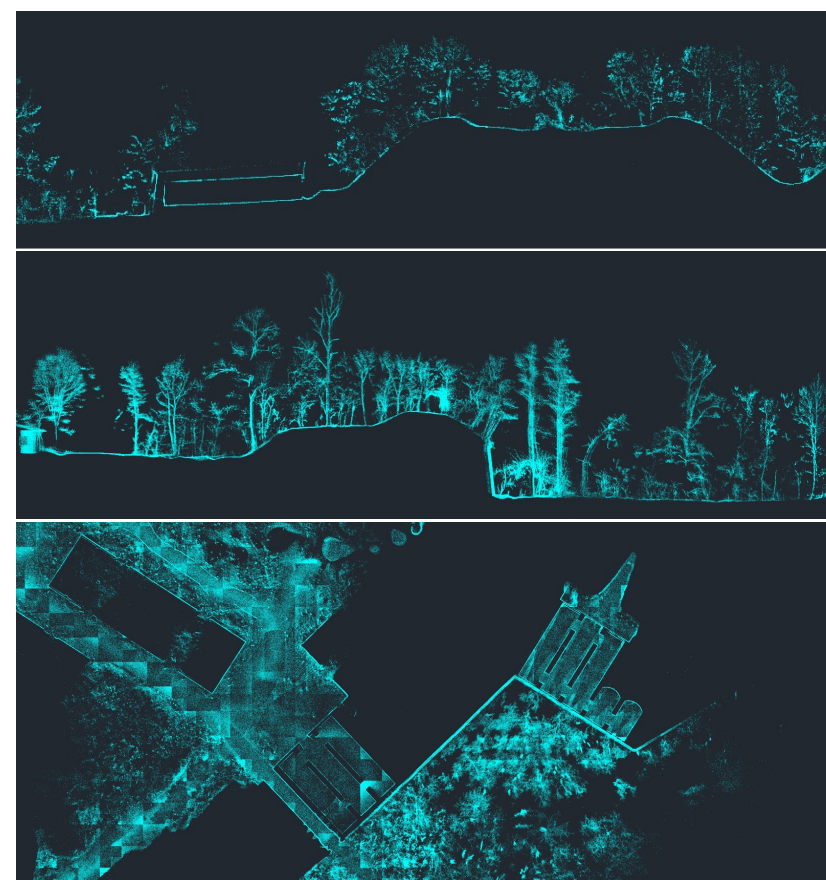

Figure 8. Cross-section of the registered point cloud (top and center) and plan view (bottom).

\subsection{D drawings and DTM}

The final point cloud was used to digitalize both the 2D drawings such as the plan of the fort core area and significant cross-sections (Figure 9) and the DTM (Figure 10). All the data used for the digitalization were produced using the Pegasus Backpack.

\section{CONCLUSIONS}

The paper aimed at testing the accuracy achievable with the Leica Pegasus Backpack MMS under a challenging scenario. The author's goal was to investigate whether present-day MMS commercial solutions could be used for big scale architectonic application in substitution to many different instruments to ease the survey practice. The results of the test show how the Pegasus Backpack can produce complete and high-resolution DTMs together with the survey of architectonic structures, both indoor and outdoor. The backpack could record data and compute the system trajectory also under the vegetation, and although to achieve 1:500 accuracy an additional survey with the total station was required, it is safe to say that under the non-extreme condition the sole MMS can produce 1:200 accuracy results for structure and environment surveys at ones. Use of the backpack is a valid and cost- and time-effective alternative for DTM extraction, especially if the high ground resolution is required.
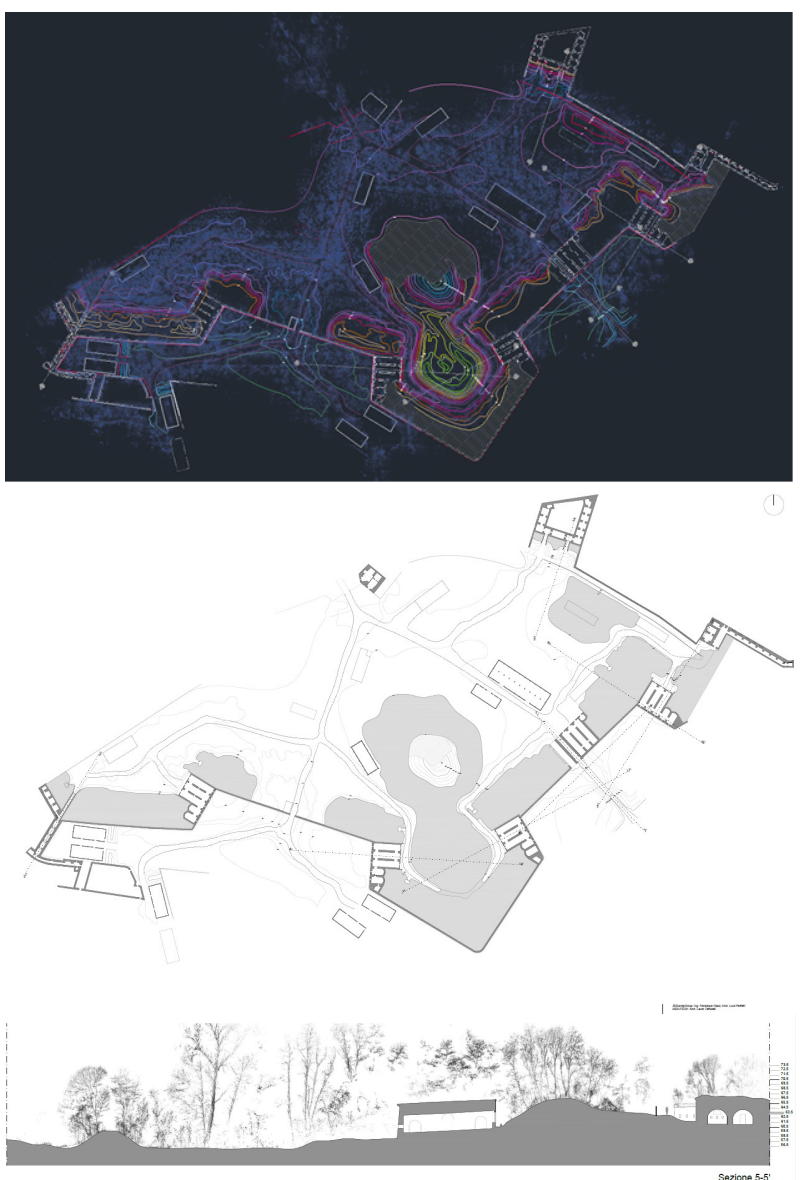

Figure 9. overlay of the Pegasus Backpack point cloud and the digitalization (top), 1:500 plan of the fort and a cross-section (bottom).

\section{ACKNOWLEDGMENTS}

The authors would like to thank Simoni Oppici and Marco Formentini of Leica Geosystems for making this research work possible, for their time spent on survey and data processing. A heartfelt thanks also to Laura Taffurelli and Fausta Fiorillo for the help provided during the field survey. 


\section{REFERENCES}

Lauterbach, H., Borrmann, D., Heß, R., Eck, D., Schilling, K., Nüchter, A., 2015. Evaluation of a Backpack-Mounted 3D Mobile Scanning System. In: Remote Sensing, 7 (10), pp.13753-13781, https://doi.org/10.3390/rs71013753.

Lehtola, V., Kaartinen, H., Nüchter, A., Kaijaluoto, R., Kukko, A., Litkey, P., ... Hyyppä, J., 2017. Comparison of the Selected State-Of-The-Art 3D Indoor Scanning and Point Cloud Generation Methods. In: Remote Sensing, 9(8), pp.796, https://doi.org/10.3390/rs9080796.

Liang, X., Kankare, V., Hyyppä, J., Wang, Y., Kukko, A., Haggrén, H., ... Vastaranta, M., 2016. Terrestrial laser scanning in forest inventories. In: ISPRS Journal of Photogrammetry and Remote Sensing, Volume 115, pp. 63-77,

https://doi.org/10.1016/j.isprsjprs.2016.01.006.

Masiero, A., Fissore, F., Guarnieri, A., Piragnolo, M., Vettore, A., 2017. Comparison of low cost photogrammetric survey with TLS and Leica Pegasus Backpack 3d models. In: The International Archives of the Photogrammetry, Remote Sensing and Spatial Information Sciences, XLII-2/W8, pp.147-153, https://doi.org/10.5194/isprs-archives-XLII-2-W8-147-2017.

Mandelli, A., Fassi, F., Perfetti, L., Polari, C., 2017. Testing different survey techniques to model architectonic narrow spaces. In: The International Archives of the Photogrammetry, Remote Sensing and Spatial Information Sciences, XLII-2/W5, pp. 505-511, https://doi.org/10.5194/isprs-archives-xlii-2-w5505-2017.

Nocerino, E., Menna, F., Remondino, F., Toschi, I., RodríguezGonzálvez, P., 2017. Investigation of indoor and outdoor performance of two portable mobile mapping systems. In: $F$.
Remondino \& M. R. Shortis (Eds.), Videometrics, Range Imaging, and Applications XIV, SPIE,

https://doi.org/10.1117/12.2270761.

Puente, I., González-Jorge, H., Martínez-Sánchez, J., Arias, P., 2013. Review of mobile mapping and surveying technologies. In: Measurement, 46(7), pp. 2127-2145,

https://doi.org/10.1016/j.measurement.2013.03.006

Ryding, J., Williams, E., Smith, M., Eichhorn, M., 2015. Assessing handheld mobile laser scanners for forest surveys. In: Remote Sensing, 7(1), pp.1095-1111, https://doi.org/10.3390/rs70101095.

Sammartano, G., and Spanò, A., 2018. Point clouds by SLAMbased mobile mapping systems: accuracy and geometric content validation in multisensor survey and stand-alone acquisition. In: Applied Geomatics, 10(4), pp.317-339, https://doi.org/10.1007/s12518-018-0221-7.

Thomson, C., Apostolopoulos, G., Backes, D., Boehm, J., 2013. Mobile laser scanning for indoor modelling.

In: ISPRS Annals of Photogrammetry, Remote Sensing and Spatial Information Sciences, II-5/W2, pp. 289-293, https://doi.org/10.5194/isprsannals-II-5-W2-289-2013.

Toschi, I., Rodríguez-Gonzálvez, P., Remondino, F., Minto, S., Orlandini, S., Fuller, A., 2015. Accuracy evaluation of a mobile mapping system with advanced statistical methods. In: The International Archives of the Photogrammetry, Remote Sensing and Spatial Information Sciences, XL-5/W4, pp. 245-253, https://doi.org/10.5194/isprsarchives-XL-5-W4-245-2015.

Tucci, G., Visintini, D., Bonora, V., Parisi, E., 2018. Examination of Indoor Mobile Mapping Systems in a Diversified Internal/External Test Field. In: Applied Sciences, 8(3), pp.401, https://doi.org/10.3390/app8030401.

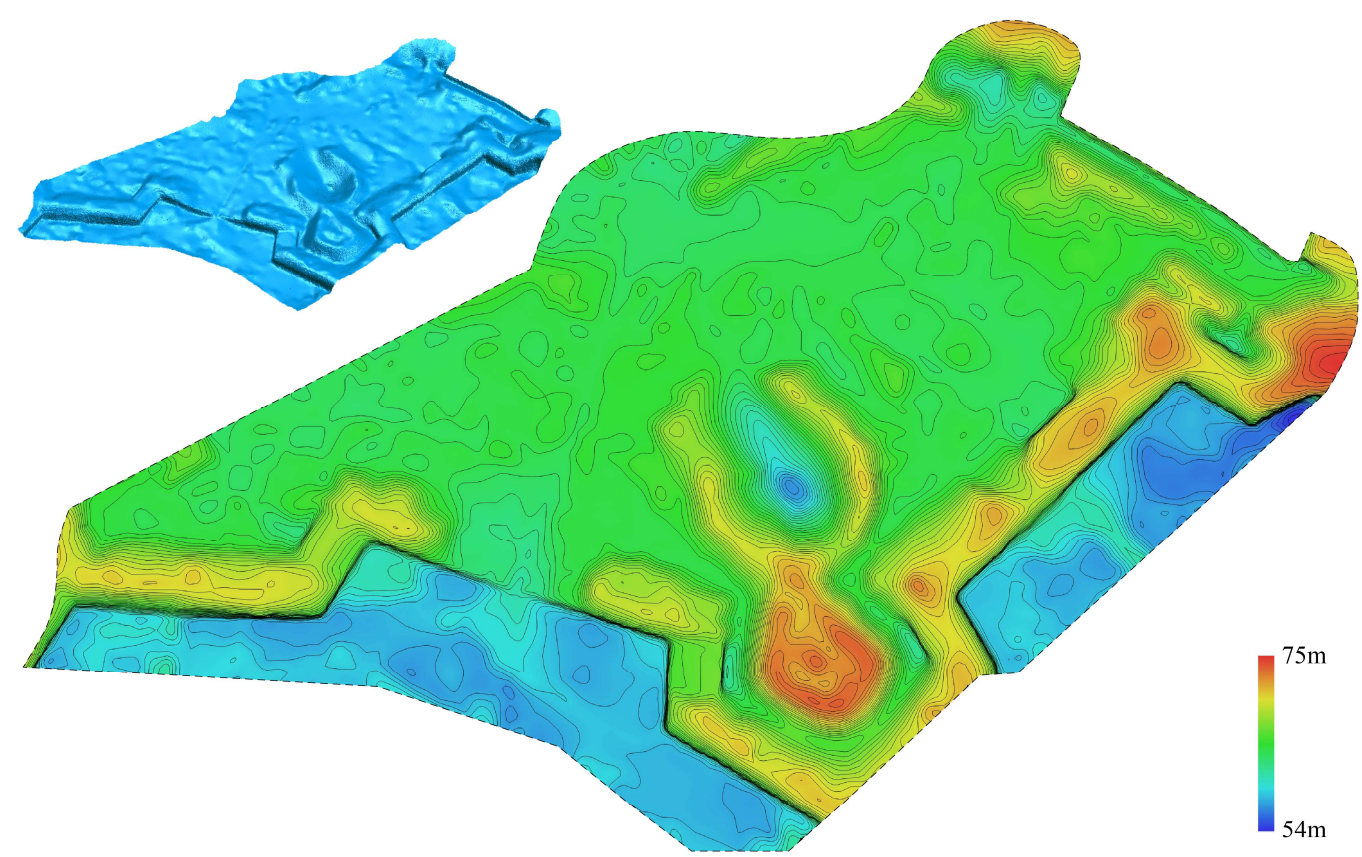

Figure 10. DTM of the Fort of Pietole obtained from the Pegasus Backpack point cloud. 